\title{
ASO Author Reflections: Gallbladder Cancer Research, "One for All and All for One” Strategy to Improve Research
}

\author{
Eduardo A. Vega, $\mathrm{MD}^{1,2}$, Timothy E. Newhook, $\mathrm{MD}^{2}$, and Jean-Nicolas Vauthey, MD, FACS ${ }^{2}$ \\ ${ }^{1}$ Department of Surgery, St. Elizabeth's Medical Center, Tufts University School of Medicine, Boston, MA; ${ }^{2}$ Department \\ of Surgical Oncology, The University of Texas MD Anderson Cancer Center, Houston, TX
}

"All for one and one for all, united we stand, divided we fall" - The Three Musketeers by Alexandre Dumas.

\section{PAST}

Although gallbladder cancer (GBC) is a relatively uncommon neoplasm in the USA and Europe, its incidence shows significant geographic variations; in reality, GBC is the most common type of biliary tract cancer worldwide. Given this scenario, progress in treatment of GBC is especially dependent on collaboration through both clinical trials and careful retrospective data analysis. Using the latter strategy, during the past 5 years, our group has been able to make significant contributions to the "orphan field" of gallbladder cancer. Examples of these contributions are the new classification of the T2 subcategory, ${ }^{1}$ the predictive role of the cystic duct lymph node, ${ }^{2}$ the detrimental effect of index cholecystectomy in $\mathrm{T} 2 \mathrm{~b} \mathrm{GBC}^{3},{ }^{3}$ and the oncologic outcomes of laparoscopic resection for incidental GBC. ${ }^{4}$

Throughout these collaborations we recognized two major problems: (1) the lack of prospective trials in gallbladder cancer, which are challenging owing to the rarity of the disease; and (2) the lack of data to guide surveillance following oncologic resection for GBC. Indeed, the appropriate follow-up and imaging schedule after oncologic resection for GBC is currently determined largely by

(C) Society of Surgical Oncology 2021

First Received: 30 December 2020

Accepted: 30 December 2020;

Published Online: 14 March 2021

J.-N. Vauthey, MD, FACS

e-mail: jvauthey@mdanderson.org physician bias and training, and can sometimes be influenced by external factors, including payer policies regarding imaging frequency, and patient factors such as anxiety and fear associated with surveillance scans.

With this in mind, a new collaborative effort was created using conditional recurrence-free survival (RFS) to inform surveillance strategy and identify factors affecting conditional RFS after oncologic extended resection (OER) for GBC.

\section{PRESENT}

In this study, ${ }^{5}$ we reviewed patients who underwent curative-intent surgery for GBC at five centers in four countries. We found that the risk of recurrence peaked at 8 months, with $84 \%$ of recurrences seen within the first 18 months of follow-up. T3-T4 disease was independently associated with risk of recurrence up to 24 months recurrence free (HR 2.71, 95\% CI 1.11-6.62, $P=0.029$ ). In addition, the study showed that recurrence rates in patients with advanced (stage III-IV) and early (stage I-II) GBC become similar starting at 36 months of follow-up. These findings suggest that the recurrence risk stratified by early and advanced stage of GBC allows for more personalized counseling of patients regarding prognosis and follow-up. Based on our study, we suggest that follow-up after OER for GBC should differ in patients with stage I-II versus stage III-IV disease. Specifically, the intensity of follow-up may be changed based on conditional RFS, with closer surveillance allocated to patients with stage III-IV disease. Of course, in the event of disease relapse, full reevaluation and deviation from any scheduled surveillance should occur. 


\section{FUTURE}

Because patients with stage III-IV disease have a high likelihood of recurrence during the first year, approximately $6 \times$ more than stage I-II disease, resection alone might be insufficient for these patients, and effective chemotherapy and other local therapy should be considered. Unfortunately, in the last decade only three randomized phase III clinical trials (ABC-02, PRODIGE12/ACCORD-18, and BILCAP), and one single-arm phase II trial (SWOG0809) have reported on the use of chemotherapy for gallbladder cancer with contradictory results. ${ }^{6}$ These contradictory findings regarding the benefit of perioperative chemotherapy for biliary tract cancer have prompted researchers to investigate novel treatments for GBC. Our group recently presented at the American Association for Cancer Research (ACCR) meeting on the immune profile and its association with cancer genomic alterations of 97 patients with GBC from the USA and Chile. $^{7}$ However, the immunological landscape of gallbladder adenocarcinoma is still far from been fully understood.

In this context, data from clinical trial EA2197 (regarding Optimal Perioperative Therapy for Incidental Gallbladder Cancer (OPT-IN) [ClinicalTrials.gov identifier: NCT04559139]: A randomized phase II/III trial) led by a collaborative effort of recognized hepatobiliary centers throughout the USA will be of utmost importance. This trial compares neoadjuvant gemcitabine and cisplatin followed by surgery plus adjuvant gemcitabine and cisplatin versus up-front surgery followed by adjuvant gemcitabine and cisplatin.

The COVID-19 pandemic is a reminder of the potential vast resources of global collaboration and innovation in medicine. We will continue to apply the lessons of collaboration towards making real progress in our field with our current and future studies, and like the classic characters of Alexandre Dumas's novel, we likewise proclaim, "all for one and one for all..."

DISCLOSURE None of the authors declare any conflicts of interest.

\section{REFERENCES}

1. Shindoh J, De Aretxabala X, Aloia TA, et al. Tumor location is a strong predictor of tumor progression and survival in $\mathrm{T} 2$ gallbladder cancer: an international multicenter study. Ann Surg. 2015;261(4):733.

2. Vega EA, Vinuela E, Yamashita S, et al. Extended lymphadenectomy is required for incidental gallbladder cancer independent of cystic duct lymph node status. J Gastrointest Surg. 2018;22(1):43-51.

3. Vega EA, Vinuela E, Okuno $\mathrm{M}$, et al. Incidental versus nonincidental gallbladder cancer: index cholecystectomy before oncologic re-resection negatively impacts survival in $\mathrm{T} 2 \mathrm{~b}$ tumors. HPB. 2019;21(8):1046-56.

4. Vega EA, De Aretxabala X, Qiao W, et al. Comparison of oncological outcomes after open and laparoscopic re-resection of incidental gallbladder cancer. Br J Surg. 2020;107(3):289-300.

5. Vega EA, Newhook TE, Kawaguchi Y, et al. Conditional recurrence-free survival after oncologic extended resection for gallbladder cancer: an international multicenter analysis. Ann Surg Oncol. 2021. https://doi.org/10.1245/s10434-021-09626-3.

6. Gamboa AC, Maithel SK. The landmark series: gallbladder cancer. Ann Surg Oncol. 2020;27(8):2846-58.

7. Carapeto FCL, Soto LMS, Foo WC, et al. Immunological and mutational landscape of gallbladder adenocarcinoma. AACR; 2020.

Publisher's Note Springer Nature remains neutral with regard to jurisdictional claims in published maps and institutional affiliations. 